\title{
Mitos y realidades de la anticoncepción hormonal combinada
}

\section{Myths and realities of combined hormonal contraception.}

\author{
Ignacio Cristóbal-García, ${ }^{1,2,3}$ José Luis Neyro-Bilbao, ${ }^{3}$ Marina Carrascoso ${ }^{1}$
}

\section{Resumen}

ANTECEDENTES: La anticoncepción ha cambiado el devenir histórico de muchas mujeres, además de permitir su desarrollo personal, sin ligarse a los embarazos sucesivos sin planificación. Hace muchas décadas que la eficacia clínica de la anticoncepción hormonal está fuera de duda, incluso por parte de la población general. Persisten, sin embargo, demasiados mitos acerca de su seguridad y sobre determinadas circunstancias de su empleo que condicionan las decisiones profesionales de la salud y de usuarias potenciales.

METODOLOGíA: Ensayo retrospectivo consistente en la búsqueda de artículos originales y revisiones sistemáticas publicados en los últimos diez años en la base de datos de PubMed, específicamente relacionados con temas de anticoncepción hormonal. Las palabras de búsqueda (MeSH) fueron: hormonal contraception, oral contraceptives, myths about oral contraceptives y otros. Se seleccionaron los artículos de mayor nivel de evidencia; se analizaron más de 50 documentos y, de éstos, se ubicaron los más relevantes en cuanto a los mitos y las realidades en torno a la anticoncepción hormonal.

CONCLUSIONES: A pesar del cambio personal y social que la anticoncepción hormonal ha supuesto en los pasados 50 años, persisten aún demasiados mitos acerca de su seguridad y relación con los sangrados regulares, que condicionan negativamente la información proporcionada a las posibles usuarias y las decisiones de la mujer que requiere anticoncepción segura y confortable.

PALABRAS CLAVE: Anticoncepción; embarazos; eficacia clínica; anticoncepción hormonal; anticonceptivos orales.

Abstract

BACKGROUND: Contraception has changed the historical development of many women and allowed personal development not linked to the attachment of successive pregnancies without planning. Many decades ago the clinical efficacy of hormonal contraception is beyond doubt even by general population. However, there are still too many myths about their safety and about certain circumstances of their use that condition the decisions of both health professionals and potential users.

METHODOLOGY: A retrospective trial consisting of the search of original articles and systematic reviews published in the last ten years in the PubMed database, specifically related to hormonal contraception. Search words (MeSH) were: hormonal contraception, oral contraceptives, myths about oral contraceptives and others. The articles with the highest level of evidence were selected, more than 50 documents were analyzed and the most relevant ones were located regarding the myths and realities around hormonal contraception.

CONCLUSIONS: Despite the personal and social change that hormonal contraception has brought about in the past fifty years, there are still too many myths about their safety and their relationship with regular bleeding, which adversely affect the information provided to potential users and decisions of the woman who needs safe and comfortable contraception.

KEYWORDS: Contraception; Pregnancies; Clinical efficacy; Hormonal contraception; Oral contraceptives.

\footnotetext{
${ }^{1}$ Servicio de Ginecología y Obstetricia, Hospital La Zarzuela, Madrid.

2 Universidad Francisco de Vitoria, Madrid, España.

${ }^{3}$ Servicio de Ginecología y Obstetricia, Universidad del País Vasco, Hospital Universitario Cruces, Baracaldo, España.
}

Recibido: agosto 2018

Aceptado: enero 2019

Correspondencia José Luis Neyro doctor@neyro.com

Este artículo debe citarse como Cristóbal-García I, Neyro-Bilbao JL, Carrascoso M. Mitos y realidades de la anticoncepción hormonal combinada. Ginecol Obstet Mex 2020;88(Supl 1):S96-S108.

https://doi.org/10.24245/gom.v88iSupl1.3854 


\section{ANTECEDENTES}

Dos de las aportaciones más importantes de la ginecología en general a la sociedad del siglo pasado tienen que ver con la separación entre el ejercicio de la sexualidad humana y su fertilidad. De una parte, se consiguió el ejercicio de la fertilidad sin sexo a través de la reproducción asistida, hace ya 40 años; ${ }^{1}$ por la otra, la anticoncepción consiguió de forma estable y voluntaria (seguramente no por primera vez, pero sí con eficacia notable), el ejercicio de la sexualidad sin fertilidad. De ello se cumplen ahora los primeros 60 años. $^{2}$

Desde siempre, la "planificación familiar" es una preocupación frecuente de las parejas, sobre todo de las jóvenes en edad reproductiva. El hecho de evitar un embarazo no deseado se relaciona con una vida sexual sana en pareja. Como todo hecho que implica una preocupación, lleva en muchos casos a la búsqueda de información, a veces sin verificar cuál es la seguridad de los datos conseguidos, sin analizar a veces la fiabilidad de la fuente.

En los últimos años se ha documentado que el origen de Internet se debe a ARPANET (Advanced Research Projects Agency Network), que originalmente era una red de computadoras del Ministerio de Defensa de Estados Unidos que propició el surgimiento de Internet en un proyecto militar que buscaba, en origen, crear una red de computadoras que uniera los centros de investigación de defensa en caso de ataques exteriores y que siguieran funcionando a pesar de que alguno de sus nodos fuera destruido. ${ }^{3}$ En la era de internet, con cantidad de información al alcance de cualquiera, aparece un problema en el ámbito de la salud: enfrentar multitud de información no verificada ni contrastada. Sin embargo, en muchos casos, la información errónea se vuelve más arraigada y menos fiel a la verdad conforme se comparte, a veces, masivamente.
Comprobar si estamos ante información fiable no siempre es fácil y, en muchos casos, implica mucho tiempo y no menos esfuerzo personal.

Desde el punto de vista etimológico, mito proviene del griego mythos, que significa relato o historia; "narración maravillosa situada fuera del tiempo histórico y protagonizada por personajes de carácter divino o heroico". ${ }^{4}$ Entendiendo mito como una "historia imaginaria que altera las verdaderas cualidades de una persona o de una cosa y les da más valor del que tienen en realidad" ${ }^{\prime \prime} y$, en este contexto, tenemos en materia de anticoncepción falsas verdades o mitos que están Ilegando a las pacientes por esta amplia vía que es internet y las redes sociales y, acaso condicionando (no siempre en positivo) la elección de su método anticonceptivo.

Y es que en temas tan sensibles como su empleo dentro de la sexualidad y su ejercicio sin fertilidad, surgen muchos mitos que se van extendiendo y pueden perjudicar la información médica más veraz y basada en la mejor evidencia científica; en lo que respecta a los anticonceptivos reversibles de larga duración son variados e incluyen verdaderos despropósitos ${ }^{6}$ y quizá, también, en temas relacionados.

A pesar de contar con métodos anticonceptivos altamente eficaces, según los datos aportados por la encuesta de anticoncepción de la Sociedad Española de Contracepción (SEC) de 2016, un $24.7 \%$ de las mujeres españolas no utiliza ningún método anticonceptivo, a pesar de que $16.4 \%$ de ellas afirma mantener relaciones sexuales; ${ }^{7}$ uno de los motivos para no emplearlos son los mitos de los probables efectos secundarios.

Entre los métodos anticonceptivos disponibles, la anticoncepción hormonal es uno de los más utilizados; es el primero en Europa y el segundo en España, donde es el método de elección de $26.8 \%$ de las usuarias, solo por detrás del pre- 
servativo. ${ }^{7}$ En España los utiliza $37.3 \%$ de las jóvenes de 20 a 24 años y $34 \%$ de las mujeres entre 25 y 29 años. $^{7}$ Si estas cifras se unen al dato conocido de que al menos $28 \%$ de las adolescentes recurre a Internet como fuente de investigación en los aspectos anticonceptivos, ${ }^{8}$ aunque la información refuerza algunos mitos lejos de aportar hechos reales, ${ }^{9}$ podremos suponer que el panorama, lejos de mejorar con toda la información y la mejor evidencia científica disponible, dista de proporcionar el mejor marco para una elección responsable de la mejor opción en métodos anticonceptivos a una gran parte de nuestra población.

El objetivo de esta revisión fue: reunir los mitos más relevantes acerca de los métodos anticonceptivos hormonales y, más concretamente, de la anticoncepción hormonal combinada, enfrentando los más comunes de todos ellos con la mejor evidencia científica y ofrecer a los profesionales implicados en la salud de la mujer y la planificación familiar información precisa para debatir con las pacientes las diferentes opciones anticonceptivas y, así, proporcionar la mejor calidad en la información anticonceptiva.

\section{PRIMER MITO}

\section{La anticoncepción hormonal combinada} aumenta el riesgo de padecer cáncer

Uno de los principales puntos de estudio desde el lanzamiento de la primera píldora anticonceptiva ha sido su seguridad, en especial por la posible relación entre la administración de hormonas exógenas y el cáncer en distintas localizaciones del organismo.

\section{Cáncer de mama}

Puesto que los anticonceptivos combinados son, finalmente, un tratamiento hormonal se ha pensado en su posible relación con los cánceres hormono-sensibles. Y es que ha sido ampliamente estudiada la relación entre el cáncer de mama y los factores hormonales con resultados contradictorios a lo largo del tiempo, por lo que es fácil pensar en una asociación con la anticoncepción hormonal.

Los estudios observacionales han sido la principal fuente de evidencia, limitada sobre todo a las preparaciones combinadas de estrógeno y progestágeno. Los estudios efectuados entre 1980 y 1990 encontraron un aumento pequeño, alrededor de $20 \%$, en el riesgo relativo de padecer cáncer de mama entre usuarias de anticonceptivos hormonales combinados. La traducción en número absoluto de casos excesivos ha sido exigua porque la prevalencia de la enfermedad es relativamente pequeña en mujeres premenopáusicas. Además, el riesgo pareció desaparecer lentamente después de 5-10 años de uso. ${ }^{10}$

Varios estudios de los últimos años han concluido que existe un ligero aumento en el riesgo relativo de cáncer de mama en mujeres premenopáusicas usuarias de anticonceptivos hormonales combinados en comparación con mujeres nunca usuarias y que este riesgo aumentó, proporcionalmente, con mayor tiempo de empleo; sin embargo, los aumentos absolutos en el riesgo fueron muy pequeños. ${ }^{11,12} \mathrm{El}$ más reciente de estos estudios notificó un riesgo relativo de cáncer de mama de $20 \%$ superior entre las usuarias de anticonceptivos hormonales (anticoncepción hormonal combinada) (RR: 1.20; IC95\%: 1.14-1.26); el riesgo relativo se elevó de 1.09 (IC95\%: 0.96-1.23) con menos de un año de uso hasta 1.38 (IC95\%: 1.26-1.51) con más de 10 años de uso, pero el aumento absoluto fue pequeño pues solo suponía 1 caso adicional por cada 7690 usuarias de anticonceptivos hormonales combinados. ${ }^{12}$ Entran en juego numerosos factores de confusión como: 
la paridad, la lactancia materna, la edad hasta el primer hijo, por lo que esta asociación debe interpretarse con mucha precaución.

Casi a renglón seguido de estas publicaciones, el American College of Obstetrics and Gynecology (ACOG) publicó unas recomendaciones para la práctica médica con una breve declaración enfocada en la que se urgía a los médicos a explicar el riesgo identificado recientemente en el contexto de los beneficios y las alternativas a los anticonceptivos hormonales, de modo que los médicos y las pacientes puedan tomar decisiones compartidas acerca de qué hacer a continuación. ${ }^{13}$ Los resultados clave de esas recomendaciones fueron las siguientes y se trascriben textuales (aun señalando que no fue posible realizar el ajuste estadístico por la edad en el momento de la menarquia, la lactancia, el consumo de alcohol ni la actividad física de las participantes en el seguimiento):

\section{El riesgo global de cáncer de mama es bajo entre usuarias de anticoncepción hormonal combinada}

Puesto que los resultados del estudio mencionado $^{14}$ acerca de los anticonceptivos con progestinas no eran congruentes, es necesario continuar los estudios acerca de sus riesgos de cáncer de mama (recuérdese que existen anticonceptivos hormonales que combinan estrógenos más progestinas y gestágenos y anticonceptivos con solo progestinas -CSP CSG-, en los que la ausencia del estrógeno puede resultar definitivamente importante a estos efectos de medir su influencia remota en el riesgo relativo de padecer cáncer de mama.

Los anticonceptivos hormonales combinados producen beneficios significativos en salud, además de anticoncepción, entre los que se incluye el menor riesgo de cáncer de ovario (CO), de endometrio (CE) y de colon (CC).
El riesgo de mortalidad por un embarazo (26.4 por cada 100,000 en 2014) es el doble del riesgo de cáncer de mama.

El estudio publicado ${ }^{14}$ tiene limitaciones debido a su incapacidad de realizar el ajuste por los posibles (y múltiples) factores de confusión.

Debería asesorarse a las mujeres acerca de los métodos anticonceptivos no hormonales y de lo que pueden hacer para reducir el riesgo de cáncer de mama (lactancia, ejercicio y limitación del alcohol).

\section{Cáncer de cuello uterino}

La infección por virus del papiloma humano (VPH) genotipos oncogénicos es condición necesaria para padecer cáncer de cuello uterino, pero otros factores de riesgo interactúan con él para definir el riesgo individual. Entre todos estos se ha relacionado al uso de anticonceptivos hormonales combinados.

La anticoncepción hormonal oral ha demostrado que el uso actual o reciente a largo plazo (más de cinco años) se relaciona con riesgo de cáncer de cuello uterino. Sin embargo, existen numerosos factores de confusión, como el hábito tabáquico, las lesiones premalignas sufridas previamente $y$, sobre todo, la necesaria infección previa por $\mathrm{VPH}$. Debe tenerse en cuenta, además, que el riesgo de cáncer de cuello uterino asociado con la anticoncepción hormonal combinada disminuye al abandonarlos hasta igualarse con la población general luego de 10 años. ${ }^{15}$

Por último, el riesgo asociado al cáncer de mama y cáncer de cuello uterino habría que ponderarlo con el efecto de la anticoncepción hormonal de disminución del riesgo de cáncer de ovario, de endometrio y de colon largamente demostrados (y no objeto de esta revisión). De hecho, determinados estudios sugieren que el efecto global del 
uso de anticonceptivos hormonales podría ser, incluso, una discreta disminución en el riesgo total de cáncer en usuarias durante cinco años o más, como se muestra con rotundidad en el Cuadro 1.16

\section{Conclusión para el primer mito}

La anticoncepción hormonal sí parece asociar un ligero aumento de riesgo tanto de cáncer de mama, más dudosamente con cáncer de cuello uterino que, sin embargo, debe interpretarse con cautela en virtud de la multitud de factores de confusión asociados y teniendo en cuenta, a su vez, su asociación con una disminución universalmente confirmada de riesgo de cánceres de ovario, endometrio y colon en el contexto de la salud general a la hora de informar a cada paciente. Además, debe recordarse al hablar de estos pormenores que el riesgo de mortalidad por un embarazo (26.4 por cada 100,000 en 2014) es el doble del riesgo de cáncer de mama. ${ }^{13}$

\section{SEGUNDO MITO}

\section{La anticoncepción hormonal combinada disminuye la fertilidad posterior.}

Uno de los mitos más arraigados en torno a la anticoncepción hormonal, sobre todo asociado con los métodos combinados por vía oral es la creencia de disminución de la fertilidad posterior al abandono del método. Puesto que es un método anticonceptivo ampliamente utilizado por usuarias jóvenes y sin deseos genésicos cumplidos, es un tema que preocupa a muchas mujeres.

Varios estudios que utilizan datos retrospectivos en el tiempo hasta la concepción, concluyen en la existencia de un retraso, a corto plazo, de 2 a 6 meses en el retorno de la fertilidad después de la utilización de métodos combinados por vía oral en comparación con otros anticonceptivos. ${ }^{17-20}$ Sin embargo, solo dos estudios han evaluado, prospectivamente, la relación entre el uso de métodos combinados por vía oral y el tiempo hasta la concepción y concluyeron que no hay asociación con cambios en la fertilidad, al menos cuando fueron dienogest o drospirenona las progestinas utilizadas en los métodos combinados por vía oral. ${ }^{21,22}$

Cuando se relata una cierta demora desde el momento de la interrupción de la anticoncepción hormonal hasta la concepción, siempre es temporal y limitada tras el cese de los anticonceptivos hormonales. ${ }^{23,24}$ En cuanto a la asociación con disminución a largo plazo de la fertilidad, numerosos estudios no evidencian tal dato, incluso, un estudio ha relacionado el uso de la anticoncepción oral a largo plazo con una mejoría de la ulterior fertilidad. ${ }^{25}$ Las tasas de concepción a los 12 meses en antiguas usuarias de anticonceptivos orales combinados, en comparación con las usuarias de dispositivos intrauterinos, preservativos y planificación familiar natural, son similares. ${ }^{23}$

Cuadro 1. Metanálisis de estudios observacionales publicados entre 2000 y 2012, que muestran los resultados de riesgo de cáncer en diversas localizaciones asociados con usuarias o nunca usuarias de anticoncepción hormonal (modificado de la cita 16)

\begin{tabular}{lc|c|c}
\hline Localización & Estudios $(\mathbf{n})$ & OR $($ IC95\%) & Incremento o descenso (\%) de RA de por vida \\
\hline Cáncer de mama & 23 & $1.08(1.00-1.17)$ & 0.89 \\
\hline Cáncer de ovario & 24 & $0.73(0-66-0.81)$ & -0.54 \\
Cáncer de endometrio & 7 & $0.57(0.43-0.77)$ & -1.77 \\
Cáncer colorrectal & 11 & $0.86(0.79-0.95)$ & -0.76
\end{tabular}

$\mathrm{OR}$, odds ratio. $\mathrm{Cl}$, intervalo de confianza. $\mathrm{RA}$ : riesgo absoluto 
Por lo tanto y hasta el momento actual, la evidencia científica no ha mostrado asociación entre la toma de anticonceptivos orales combinados y disminución de la fertilidad a largo plazo. Así pues, esta relación en ningún caso debiera informarse como negativa durante el asesoramiento anticonceptivo y tampoco debiera considerarse por parte de la usuaria ante la elección de su método anticonceptivo. En el grupo de mujeres mayores de 35 años, en las que la fertilidad natural comienza a decrecer, la información a este respecto debiera ser particularmente personalizada e individualizada, en función de las expectativas ulteriores de cada usuaria.

\section{TERCER MITO}

Los anticonceptivos hormonales combinados favorecen el aumento de peso

El aumento de peso secundario a la toma de anticonceptivos hormonales es un mito que ha perdurado en el tiempo entre usuarias e, incluso, médicos dedicados al cuidado de la salud de la mujer. Esta preocupación por el aumento de peso puede limitar el uso de este método anticonceptivo a pesar de su alta eficacia bien demostrada. Sin embargo, la relación entre anticoncepción hormonal y el aumento de peso no ha sido demostrada con suficiente evidencia científica.

Una abultada y exhaustiva revisión Cochrane (metanálisis que incluyó 49 ensayos) concluyó que la evidencia disponible era insuficiente para determinar el efecto de la anticoncepción hormonal en el peso, pero no se observó un efecto importante. Los ensayos incluyeron 85 comparaciones de cambio de peso para 52 pares de anticonceptivos distintos (o placebos). Los cuatro ensayos con un grupo placebo o ningún grupo de intervención no encontraron evidencia que respaldara una asociación causal entre la anticoncepción hormonal combinada o una combinación de parche cutáneo y cambio de peso. La mayor parte de las comparaciones de combinaciones diferentes de anticonceptivos hormonales no mostró diferencias sustanciales en el peso. Y es que, los autores continúan, los ensayos para evaluar el vínculo entre anticoncepción hormonal y el cambio de peso requieren un grupo placebo o no hormonal para controlar otros factores, incluidos los cambios en el peso a lo largo del tiempo y esto no suele ser posible en ensayos clínicos con anticonceptivos por razones evidentes. Se concluye, finalmente, que la bibliografía era insuficiente para establecer una definitiva relación entre el efecto de los anticonceptivos hormonales y el aumento de peso, pero que no existe evidencia de un efecto importante en el peso. ${ }^{26}$

En un estudio que incluyó varias naciones europeas se confirmó que las mujeres que informaban un aumento de peso ( $R R=1.4)$ tenían un riesgo mayor de suspensión del método en comparación con quienes no informaron este aumento. ${ }^{27} \mathrm{Y}$ es que la sola percepción del aumento de peso puede llevar a la interrupción del anticonceptivo. Un estudio estadounidense encontró que las mujeres que dejaron los anticonceptivos hormonales tenían más probabilidades de informar de un aumento de peso que quienes continuaban utilizándolos, incluso $\sin$ una diferencia significativa en el cambio de peso entre ambos grupos. ${ }^{28}$

Numerosos estudios han tenido como objetivo evaluar la influencia a corto y largo plazo de la anticoncepción hormonal en el peso corporal, incluso evaluando diferentes formulaciones con distintas dosis de etinilestradiol como estrógeno, el probable inductor de los cambios de peso en teoría, concluyendo que no hay diferencias significativas entre el aumento de peso y la utilización de anticonceptivos hormonales combinados, como muestra la Figura 1 en la que se aprecia que la distribución normal de las ganancias y pérdidas de peso en dos formulaciones diferentes no tiene diferencias significativas. ${ }^{29}$ 


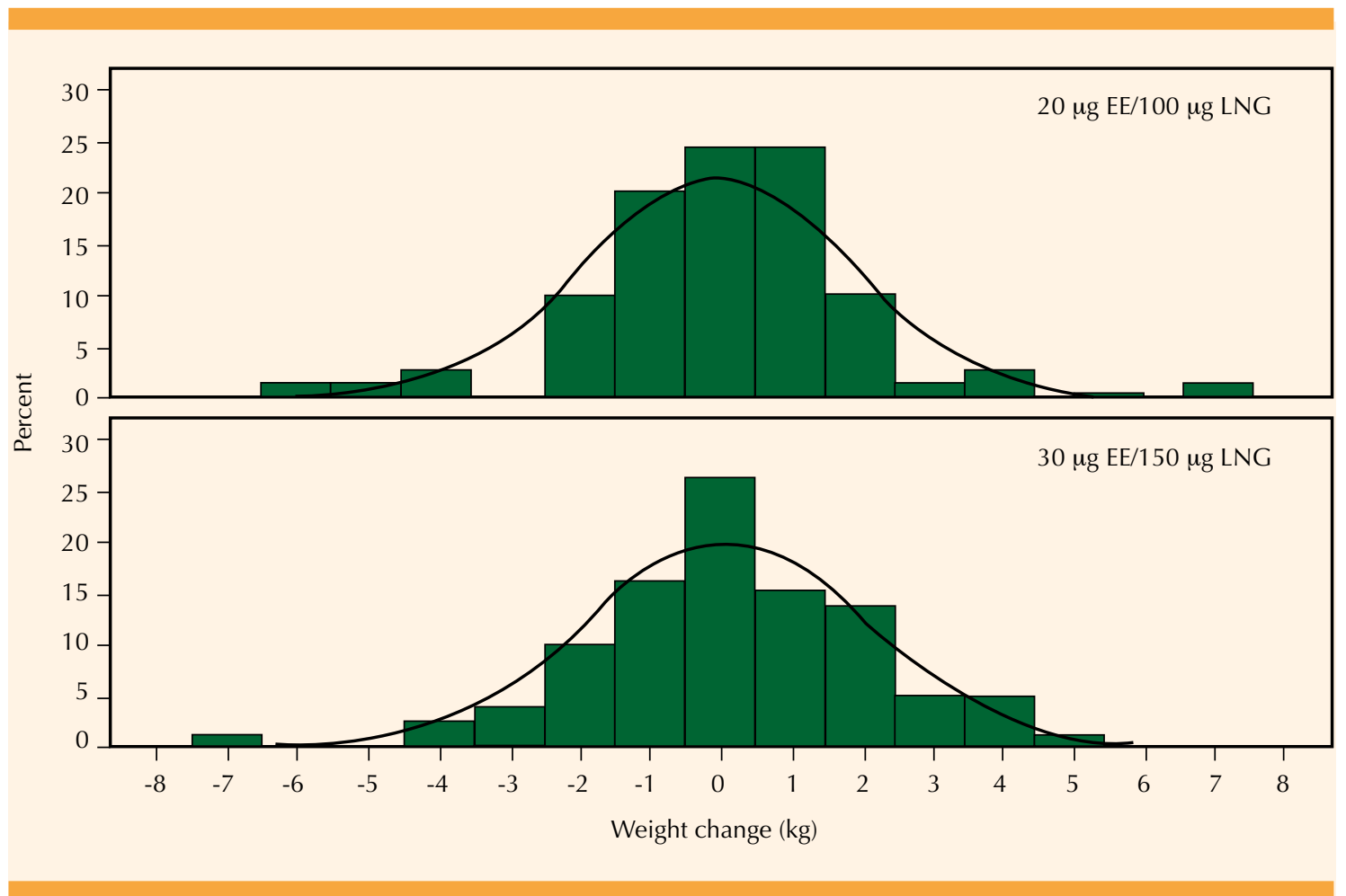

Figura 1. Cambio de peso (kg) después de tres meses de uso de anticonceptivos orales combinados, con reparto de las usuarias según la distribución normal, tanto con una dosificación de estrógeno de 20 como de 30 g de etinilestradiol con levonorgestrel (modif de la cita29).

Las hipotéticas vías fisiopatológicas a través de las que la anticoncepción hormonal combinada podría causar aumento de peso, sea en kilogramos o en índice de masa corporal, incluyen la retención de líquidos, que podría ser inducida por el efecto mineralocorticoide de etinilestradiol sobre el eje de producción de la renina-angiotensina-aldosterona con una acción antidiurética final; un aumento de grasa subcutánea mediado por la acción de los estrógenos de la combinación; un efecto en la saciedad y el apetito, lo que lleva a un aumento en la ingesta de alimentos, así como un aumento de la masa muscular mediado por andrógenos. ${ }^{29,30}$

A pesar de lo extendida de esta creencia del aumento de peso corporal que ha obligado a efectuar numerosos estudios $y$, a pesar de ser causa importante de abandono de la toma de anticonceptivos hormonales combinados, la asociación causal entre la toma de estos y su efecto positivo en el peso corporal no se ha podido demostrar, incluso con diferentes formulaciones de dosis de estrógeno.

\section{CUARTO MITO}

\section{La anticoncepción hormonal combinada necesita descansos periódicos}

Uno de los mitos más tradicionalmente extendidos y no solo entre usuarias sino también entre profesionales de la salud e, incluso, entre ginecólogos es la necesidad de descansos en la toma luego de unos meses de iniciada la anticoncepción hormonal combinada o, como norma 
periódica, después de 12 o 18 meses de empleo continuo. El asunto hacía referencia a las dosis administradas en los primeros años de empleo de anticonceptivos hormonales combinados durante los que el componente estrogénico tenía dosis varias veces superiores a las utilizadas hoy y desde hace más de 30 años, por lo que ya no existe esa "necesidad" de descansar la toma del anticonceptivo hormonal. Es digno de mención que limitar la cantidad de ciclos de píldoras puede acarrear la suspensión no deseada del método y mayor riesgo de embarazo y son circunstancias nunca deseadas cuando se inicia, precisamente, un tratamiento anticonceptivo. ${ }^{31}$

Si bien el porcentaje tiende a ir descendiendo con el paso de los años, prácticamente una de cada tres mujeres que emplea regularmente anticonceptivos hormonales combinados en España mantiene descansos periódicos, con una sugerencia que procede, masivamente (más del $70 \%$ ), del médico, de la matrona o de la enfermera que atiende sus requerimientos en esta área. ${ }^{7}$ Con todo, $20.3 \%$ de la mujeres que lleva a cabo descansos periódicos lo hace sin que se deba a una indicación médica: 16.5\% lo hace por decisión personal y $3.8 \%$ por consejo de amigas o familiares. De acuerdo con el método hormonal utilizado, el descanso es menor entre mujeres que recurren al anillo vaginal (41.2\%), seguidas de las que utilizan anticonceptivos hormonales combinados (51.2\%). ${ }^{7}$

La aparición de ciertos efectos secundarios tiene relación con el inicio del tratamiento y pueden aparecer tras cada descanso, precisamente. ${ }^{32} \mathrm{EI}$ efecto secundario más documentado y serio debido a la anticoncepción hormonal combinada es la trombosis venosa profunda y el pico de máximo riesgo de aparición está en los primeros meses luego del inicio de tratamiento; si se realizan esos descansos el reinicio del tratamiento hace que la paciente quede expuesta al riesgo de trombosis más veces. ${ }^{33}$
Numerosos estudios evalúan la seguridad del uso continuado de la anticoncepción hormonal y son muchas las ventajas de esta posología; entre ellas, la reducción de la posibilidad de ovulaciones de escape mediante tratamientos prolongados, sin que esto afecte a la recuperación del estado fértil ${ }^{34} y$, además, el efecto protector de ciertos cánceres aumenta según se incrementa el tiempo de uso..$^{15,16,35}$ Se ha anotado, por otro lado, que los (mal Ilamados) descansos contribuyen a embarazos no deseados debido a la interrupción de la pauta y la rutina que se crea con ello. ${ }^{31}$ Cuando se estudian los hechos que más asocian olvido de la toma de una píldora diaria, los descansos son uno de ellos y la mayor parte de las veces, el reinicio es una etapa de más riesgo de que se produzcan nuevos olvidos. ${ }^{36}$

Para concluir lo que respecta a los descansos periódicos por la toma continua de anticonceptivos hormonales combinados, la mejor evidencia científica disponible no justifica hacer descansos con las dosis actualmente administradas, hasta el extremo de que en este momento de la historia de la anticoncepción hormonal está totalmente desaconsejado.

\section{QUINTO MITO}

\section{Con la anticoncepción hormonal combinada se hace necesario mantener la menstruación todos los meses}

De igual manera que los descansos pasaron a la historia (moderna) de la anticoncepción hormonal, aún persiste la "necesidad" de tener sangrado mensual luego de cada ciclo de anticoncepción, muy a pesar de que en ausencia de esteroides ováricos, sea el régimen y la dosificación que sea que se indique en el tratamiento de anticoncepción hormonal, por el no funcionamiento del eje hipotálamo-hipófiso-ovárico, en todo caso sería un sangrado por deprivación y no una menstruación verdadera. ${ }^{37}$ 
Realmente, el asunto tiene que ver con el origen verdaderamente mítico de la menstruación por el que en la antigüedad, incluso, se condenaba a la impureza a la mujer durante esos días de fluido menstrual. Es más, el libro Levítico del Pentateuco señalaba, textualmente, que: "cuando una mujer tenga flujo, si el flujo en su cuerpo es sangre, ella permanecerá en su impureza menstrual por siete días; y cualquiera que la toque quedará inmundo hasta el atardecer".$^{38}$ La impureza del fluido menstrual por el desconocimiento de la fisiología femenina imperante hace 25 a 27 siglos cuando ese libro se escribió, se extendía a las relaciones sexuales pues "si un hombre se acuesta con ella y su impureza menstrual lo mancha, quedará inmundo por siete días, y toda cama sobre la que él se acueste quedará inmunda".

Pero esta visión del fluido menstrual y su mitificación, que aún arrastramos en muchas culturas, se extendió todavía seis siglos después al mundo romano del que somos herederos cultural y médicamente, pues entonces se afirmaba que "el contacto con el flujo menstrual de la mujer amarga el vino nuevo, hace que las cosechas se marchiten, mata los injertos, seca semillas en los jardines, causa que las frutas se caigan de los árboles, opaca la superficie de los espejos, embota el filo del acero y el destello del marfil, mata abejas, enmohece el hierro y el bronce, y causa un terrible mal olor en el ambiente" ${ }^{\prime 3}$

A pesar de lo distante que puede parecernos, estos mitos de la menstruación continúan, incluso, en nuestra culta Europa que, casi catorce siglos más tarde que el Levítico fuera escrito veía cómo los sabios de aquel entonces afirmaban que "esa sangre es tan abominable e impura, que a través de su contacto las frutas no maduran, las plantas se marchitan, la hierba muere, los árboles pierden su fruto, el aire se oscurece, si los perros la comen quedan afligidos de la rabia... Y las relaciones sexuales en este periodo menstrual son muy riesgosas", como afirmaba Julius Rufinus. ${ }^{40}$
Desde entonces, luego de muchos años de evolución en el aspecto social y, sobre todo, reproductivo, el número de menstruaciones que tiene una mujer a lo largo de su vida ha variado sensiblemente. En la actualidad se estima que una mujer tiene 450 ciclos menstruales de media, de forma diferenciada a cuando el embarazo era la norma y se asume que "la simplicidad misma es eliminar la menstruación con píldoras anticonceptivas orales seguras, baratas y ampliamente disponibles". ${ }^{41}$

Pero, procurando con anticonceptivos hormonales no tener la menstruación son varios estudios los que han realizado un análisis del endometrio tras varios meses sin ser "descamado", sin que se hayan identificado alteraciones o asociación con patología alguna, ni por supuesto aumento de la incidencia de hiperplasia. ${ }^{42}$ Pero, aún más, pues no menstruar todos los meses, además de no ser perjudicial para el endometrio, puede suponer diversos beneficios para la usuaria. Son bien conocidos los síntomas durante la fase menstrual, que en muchos de los casos limitan durante esos días la calidad de vida de muchas mujeres. En algunas usuarias de anticonceptivos hormonales estos síntomas aparecen en cada periodo libre de hormonas (dolor pélvico en distintos grados, cefalea, mastodinia, hinchazón general). ${ }^{43}$ Parece lógico pensar que al disminuir el número de hemorragias por deprivación hormonal, que no menstruaciones "naturales", puedan disminuir los días que la usuaria está expuesta a sufrir esos síntomas; de tal forma que si elige tener cuatro sangrados regulares al año, será menos probable que aparezcan estos síntomas, porque los cambios hormonales que los originan tienen menores variaciones en sangre en las pautas continuas o prolongadas de anticoncepción hormonal que en las pautas cíclicas clásicas. Se trata de tener menos menstruaciones y que estas sean menos sintomáticas. ${ }^{44}$

La tolerancia, el cumplimiento y la efectividad clínica son mayores en las pautas continuas o 
prolongadas que con las pautas convencionales; a pesar de todo ello, la tasa de continuidad es significativamente menor porque el patrón de sangrado con manchado inesperado es algo mayor, ${ }^{45}$ como muestra la Figura 2. Seguramente, un buen asesoramiento al respecto señalando las enormes ventajas del uso extendido podría minimizar los pequeños inconvenientes de los manchados ocasionales (también dependientes de la dosificación y de la distinta composición del anticonceptivo hormonal), enseñando a la usuaria a hacer privación por cuatro días apenas, acaso cuando el manchado aparece y se convierte en molesto luego de unos pocos días.

Está confirmada tanto la seguridad de que la toma continuada no solo no es perjudicial sino benéfica por todo lo anotado, que actualmente se dispone ya de varias pautas diferentes con distintas formulaciones, incluso, de tratamientos por 84 y hasta por 120 días seguidos tanto en Europa como en EstadosUnidos. ${ }^{46}$ Está demostrado que las pautas continuas disminuyen las cefaleas relacionadas con la menstruación y todos los síntomas dependientes de ella; $;{ }^{45,46}$ se confirman, incluso, ventajas en el tratamiento a largo plazo de un trastorno tan condicionante de la calidad de vida de las afectadas como la endometriosis, ${ }^{47}$ por solo citar algunos de los múltiples ejemplos de ventajas de las pautas continuas o extendidas.

Desde el mismo punto de vista de la seguridad, los efectos secundarios son similares a los verificados en las pautas convencionales, no observándose incremento del riesgo trombótico ni de aparición de enfermedad cardiovascular. El retorno a la fertilidad es, también, similar en ambas pautas y además de manera independiente de la formulación de cada anticonceptivo hormonal. ${ }^{48-51}$

Por lo tanto, es sencillamente falso afirmar que sea necesario sangrar de forma periódica mensualmente; es más, en todo caso, debiera informarse a la usuaria durante el asesoramiento anticonceptivo el sangrado mensual de las pautas cíclicas en ningún modo es una menstruación normal sino un sangrado por deprivación y que este puede "programarse" a voluntad (viajes, veranos, exámenes, vacaciones...). Está demostrado, incluso, que no se obtiene ningún beneficio y que, además, las pautas que provocan ciclos largos tienen ventajas añadidas que pueden mostrarse en la presentación del trata-

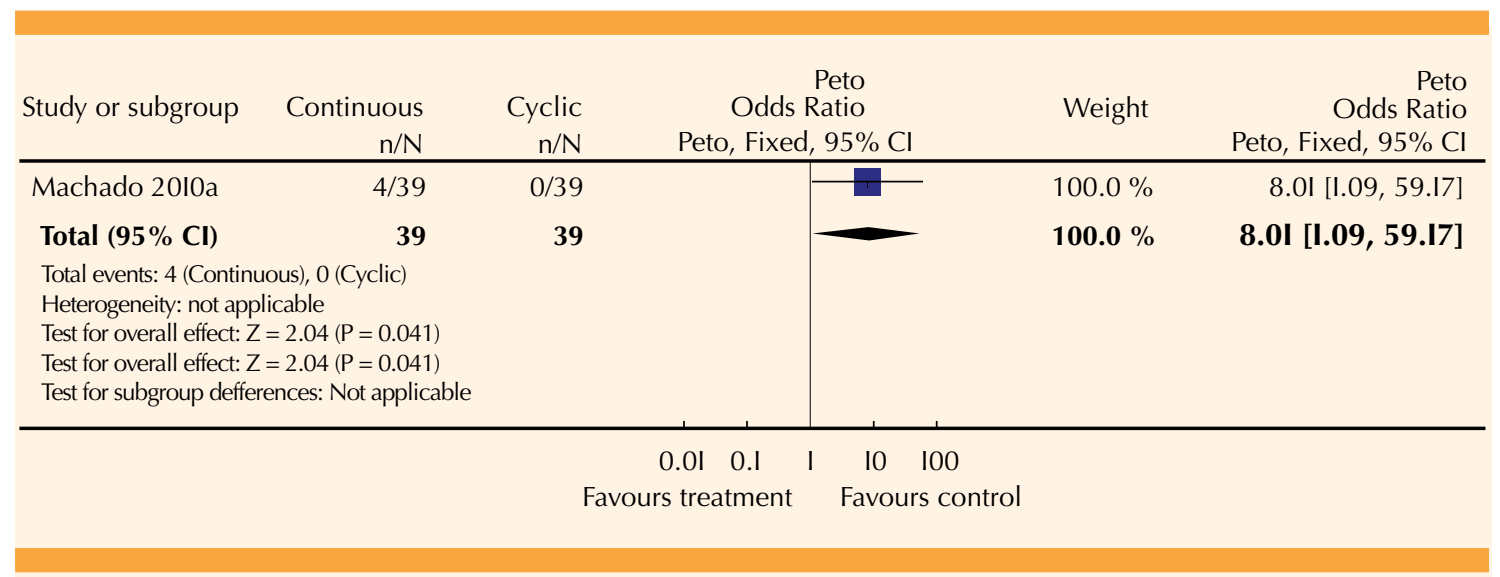

Figura 2. Resultado de la interrupción en la toma de anticonceptivos orales combinados por razones de sangrado en la comparación con $30 \mu \mathrm{g}$ de etinilestradiol y $3 \mathrm{mg}$ de drospirenona, en ciclos de 28 días versus ciclos de 168 días durante 6 meses, mostrando el significativo incremento de la misma cuando hay sangrados no esperados (modif. de la cita 45). 
miento como valores añadidos que trascienden a la anticoncepción.

\section{COMENTARIOS FINALES Y CONCLUSIONES}

Seguramente existen pocas áreas del saber médico que, acaso por su repercusión personal y sobre la vida en pareja así como también por la trascendencia social que provoca su empleo, soporten tantos mitos y falsedades como la anticoncepción hormonal. ${ }^{52}$ Asumida la eficacia anticonceptiva de los anticonceptivos hormonales por la población en general desde hace muchos años; ${ }^{7,52}$ sin embargo persisten mitos acerca de su inocuidad, seguramente basados en los acontecimientos ya históricos y derivados de la época en que la anticoncepción hormonal tenía otras dosificaciones ya abandonadas por completo hace décadas.

Tomando como base la mejor de las evidencias científicas que sobre este tema es muy abundante, podemos concluir con rotundidad que:

- La anticoncepción hormonal combinada, en general, se muestra o perfectamente neutral respecto al riesgo que su empleo provoca de padecer cáncer de mama o que apenas lo incrementa levemente luego de décadas de uso continuado.

- El riesgo de mortalidad por un embarazo, que es lo que pretende evitar la anticoncepción hormonal combinada (26.4 por 100,000 en 2014) es el doble del riesgo de cáncer de mama.

- Simultáneamente, los anticonceptivos hormonales combinados son absolutamente indiferentes frente al supuesto incremento de padecer cáncer de cuello uterino que nunca se ha demostrado.

- Existe suficiente evidencia científica que soporta la idea de que los anticonceptivos hormonales combinados provocan una significativa disminución universalmente confirmada de riesgo de cáncer de ovario, cáncer de endometrio y cáncer de colon en el contexto de la salud general.

- La evidencia científica no ha mostrado asociación entre la toma de anticonceptivos hormonales orales combinados y disminución de la fertilidad posterior a largo plazo.

- No es cierta la asociación causal entre la toma de un anticonceptivo hormonal y un efecto positivo en el peso corporal, incluso con diferentes formulaciones de dosis de estrógeno.

- La mejor evidencia científica disponible no justifica hacer descansos con las dosis actualmente administradas, por lo que deben desaconsejarse los descansos periódicos durante los tratamientos con anticonceptivos hormonales combinados.

- No solo no es necesario, sino que es sencillamente falso afirmar que sea necesario sangrar de forma periódica mensualmente, pues no se obtiene ningún beneficio $y$, además, las pautas que provocan ciclos largos de tratamiento tienen beneficios añadidos.

- Todas estas circunstancias debieran incluirse en la información que se suministra a cada usuaria potencial de anticonceptivos hormonales para su mejor conocimiento y favorecer así un más adecuado discernimiento, por parte de todos los profesionales de la salud implicados en la atención a la mejor salud de la mujer.

\section{REFERENCIAS}

Steptoe PC, et al. Birth after reimplantation of a human embryo. Lancet 1978;2:366. 
Cristóbal-García I y col. Mitos y realidades de la anticoncepción hormonal combinada

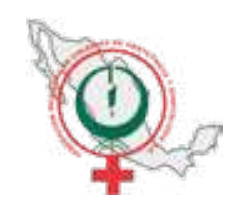

2. Pinkus $\mathrm{G}$, et al. Fertility control with oral medication. Am J Obstet Gynecol 1958;75(6):1333-46.

3. Hauben R. The Internet: On its International Origins and Collaborative Vision. 2004. http://www.ais.org/ jrh/acn/ ACn12-2.a03.txt

4. Diccionario de la Lengua Española. Edición del Tricentenario. Actualización 2017. http://dle.rae. es/?id=PQM1Wus|PQMf1C3.

5. Spanish Oxford living Dictionaries. https:// es.oxforddictionaries.com/definicion/mito.

6. Neyro JL, et al. Mitos y realidades de los anticonceptivos reversibles de larga duración. Ginecol Obstet Mex 2015;83:707-21.

7. Encuesta Sociedad Española de Contracepción 2016. http://hosting.sec.es/descargas/OBS_EncuestaAncticoncepcion2016.pdf.

8. Tolani AT, et al. Many websites fail to dispel myths about IUDs, emergency contraception, birth control and proper timing of pap smears. J Adolescent Health 2009; 44:S24S25. https://doi.org/10.1016/j.jadohealth.2008.10.056

9. Ridríguez D, et al. Problematic uses o ICT's amog young people in their personal and school life. Comunicar 2018;26(5):91-100. https://www.uoc.edu/opencms_portal2/opencms/ES/in3/publicacions/journal-papers/list.ht ml?groupCode $=$ ALL\&mask $=\&$ pubSubmit $=$.

10. Zolfaroli I, et al. Hormonal contraceptives and breast cancer: Clinical data. Eur J Obstet Gynecol Reprod Biol 2018;230:212-216. https://doi.org/10.1016/j.ejogrb.2018.03.058.

11. Anothaisintawee $\mathrm{T}$, et al. Risk factors of breast cancer: a systematic review and meta-analysis. Asia Pac J Public Health 2013;25(5):368-87. https://doi. org/10.1177/1010539513488795.

12. Mørch L, et al. Contemporary Hormonal Contraception and the Risk of Breast Cancer. Obstet Gynecol Surv 2018;73(4):215-17.

13. Mørch LS, et al. Contemporary Hormonal Contraception and the Risk of Breast Cancer. N Engl J Med 2017;377(23):222839. https://doi.org/10.1056/NEJMoa1700732

14. American College of Obstetricians and Gynecologists. Practice Advisory: Hormonal Contraception and Risk of Breast Cancer. https://www.acog.org/Clinical-Guidanceand-Publications/Practice-Advisories/Practice-AdvisoryHormonal-Contraception-and-Risk-of-Breast-Cancer.

15. La Vecchia C, et al. Oral contraceptives, human papillomavirus and cervical cancer. Eur J Cancer Prev 2014;23(2):11012. https://doi.org/10.1097/CEJ.0000000000000000.

16. Bassuk $\mathrm{S}$, et al. Oral contraceptives and menopausal hormone therapy: relative and attributable risks of cardiovascular disease, cancer, and other health outcomes. Ann Epidemiol 2015;25(3):193-200. https://doi.org/10.1016/j. annepidem.2014.11.004.

17. Vessey MP, et al. Fertility after stopping different methods of contraception. Br Med J 1978;1(6108):265-7.
18. Hassan MA, et al. Is previous use of hormonal contraception associated with a detrimental effect on subsequent fecundity? Hum Reprod 2004;19(2):344-51.

19. Kaplan B, et al. Use of various contraceptive methods and time of conception in a community-based population. Eur J Obstet Gynecol Reprod Biol 2005;123(1):72-6. https://doi. org/10.1016/j.ejogrb.2005.06.033

20. Axmon A, et al. Factors affecting time to pregnancy. Hum Reprod 2006;21(5):1279-84. https://doi.org/10.1093/ humrep/dei469

21. Wiegratz I, et al. Fertility after discontinuation of treatment with an oral contraceptive containing 30 microg of ethinyl estradiol and $2 \mathrm{mg}$ of dienogest. Fertil Steril 2006;85(6):1812-9. https://doi.org/10.1016/j.fertnstert.2005.11.052

22. Cronin $\mathrm{M}$, et al. Rate of pregnancy after using drospirenone and other progestin-containing oral contraceptives. Obstet Gynecol 2009;114(3):616-22. https://doi.org/10.1097/ AOG.0b013e3181b46f54.

23. Barnhart $\mathrm{KT}$, et al. Return to fertility following discontinuation of oral contraceptives. Fertil Steril 2009;91(3):659-63. https://doi.org/10.1016/j.fertnstert.2009.01.003.

24. Mikkelsen E, et al. Pre-gravid oral contraceptive use and time to pregnancy: a Danish prospective cohort study. Hum Reprod 2013;28(5):1398-405. https://doi.org/10.1093/ humrep/det023.

25. Farrow A, et al. Prolonged use of oral contraception before a planned pregnancy is associated with a decreased risk of delayed conception. Hum Reprod 2002;17(11):3009-10.

26. Gallo MF, et al. Combination contraceptives: effects on weight. Cochrane Database Syst Rev 2014;29;1:CD003987. https://doi.org/10.1002/14651858.CD003987.pub5.

27. Rosenberg MJ, et al. Use and misuse of oral contraceptives: risk indicators for poor pill taking and discontinuation. Contraception 1995;51(5):283-8.

28. Emans SJ, et al. Adolescents' compliance with the use of oral contraceptives. JAMA 1987;257(24):3377-81.

29. Mayeda E, et al. Weight and body composition changes during oral contraceptive use in obese and normal weight women. J Womens Health (Larchmt) 2014;23(1):38-43. https://doi.org/10.1089/jwh.2012.4241.

30. Lindh I, et al. The long-term influence of combined oral contraceptives on body weight. Hum Reprod 2011;26(7):191724. https://doi.org/10.1093/humrep/der094.

31. Organización Mundial de la Salud OMS. Recomendaciones sobre prácticas seleccionadas para el uso de anticonceptivos, tercera edición [Selected practice recommendations for contraceptive use, $3^{\text {rd }}$ edition] ISBN 978-92-4-356540-8. 2018

32. Chabbert-Buffet $\mathrm{N}$, et al. Missed pills: frequency, reasons, consequences and solutions. Eur J Contracept Reprod Health Care 2017;22(3):165-69. https://doi.org/10.1080 /13625187.2017.1295437.

33. Sitruk-Wae R. Hormonal contraception and thrombosis. Fertil Steril. 2016;106(6):1289-94. https://doi. org/10.1016/j.fertnstert.2016.08.039. 
34. Letourneau J, et al. Long-term hormonal contraceptive use is associated with a reversible suppression of antral follicle count and a break from hormonal contraception may improve oocyte yield. J Assist Reprod Genet 2017;34(9):113744. https://doi.org/10.1007/s10815-017-0981-8.

35. Cooper D, et al. Oral Contraceptive Pills. https://www.ncbi. nlm.nih.gov/books/NBK430882/.

36. Turner J. Current barriers and potential strategies to increase the use of long-acting reversible contraception to reduce the rate of unintended pregnancies in Australia: An expert roundtable discussion. Aust N Z J Obstet Gynaecol 2017;57(6):206-12. https://doi.org/10.1111/ajo.12587.

37. Vandever MA, et al. Evaluation of pituitary-ovarian axis suppression with three oral contraceptive regimens. Contraception. 2008;77(3):162-70. https://doi.org/10.1016/j. contraception.2007.11.005.

38. Levítico 15:19-33. https://www.biblestudytools.com/bla/ levitico/passage/?q=levitico+15:19-33.

39. Gayo Plinio Segundo. Historia Natural, libro 28, 23, 78-80.

40. Julius Rufinus Von Bologna, Summa Decretorum, passim. Accesible en https://www.theologie.uni-wuerzburg.de/ fileadmin/01040030/Rufin_Singer_fulltext.pdf.

41. Thomas SL, et al. Nuisance or natural and healthy: should monthly menstruation be optional for women? Lancet 2000;355(9207):922-24. https://doi.org/10.1016/S01406736(99)11159-0

42. Miller L, et al. Continuous combination oral contraceptive pills to eliminate withdrawal bleeding: a randomized trial. Obstet Gynecol 2003;101(4):653-61.

43. Sulak PJ, et al. Hormone withdrawal symptoms in oral contraceptive users. Obstet Gynecol 2000;95(2):261-66.

44. Willis SA, et al. Greater inhibition of the pituitary--ovarian axis in oral contraceptive regimens with a shortened hormone-free interval. Contraception 2006;74(2):100-3. https://doi.org/10.1016/j.contraception.2006.02.006

45. Edelman A, et al. Continuous or extended cycle vs. cyclic use of combined hormonal contraceptives for contraception. Cochrane Database Syst Rev 2014;29(7):CD004695. https://doi.org/10.1002/14651858.CD004695.pub3.

46. Wiegratz I, et al. Effect of extended-cycle regimen with an oral contraceptive containing $30 \mathrm{mcg}$ ethinylestradiol and $2 \mathrm{mg}$ dienogest on bleeding patterns, safety, acceptance and contraceptive efficacy. Contraception 2011;84(2):133-43. https://doi.org/10.1016/j.contraception.2011.01.002.

47. Vercellini $\mathrm{P}$, et al. Continuous use of an oral contraceptive for endometriosis-associated recurrent dysmenorrhea that does not respond to a cyclic pill regimen. Fertil Steril 2003;80:560-63.

48. Davis AR, et al. Occurrence of menses or pregnancy after cessation of a continuous oral contraceptive. Fertil Steril 2008;89(5):1059-63. https://doi.org/10.1016/j.fertnstert.2007.05.012

49. Wiegratz I, et al. Effects of conventional or extendedcycle regimen of an oral contraceptive containing $30 \mathrm{mcg}$ ethinylestradiol and $2 \mathrm{mg}$ dienogest on various hemostasis parameters. Contraception 2008;785:384-91. https://doi. org/10.1016/j.contraception.2008.06.015.

50. Machado RB, et al. Effect of a continuous regimen of contraceptive combination of ethinylestradiol and drospirenone on lipid, carbohydrate and coagulation profiles. Contraception 2010;81(2):102-6. https://doi.org/10.1016/j. contraception.2009.08.009.

51. Neyro JL, Elorriaga MA, Cristóbal I, et al. Comentario del experto en anticoncepción. Barcelona: Ediciones Médicas. www.euromedice.net.

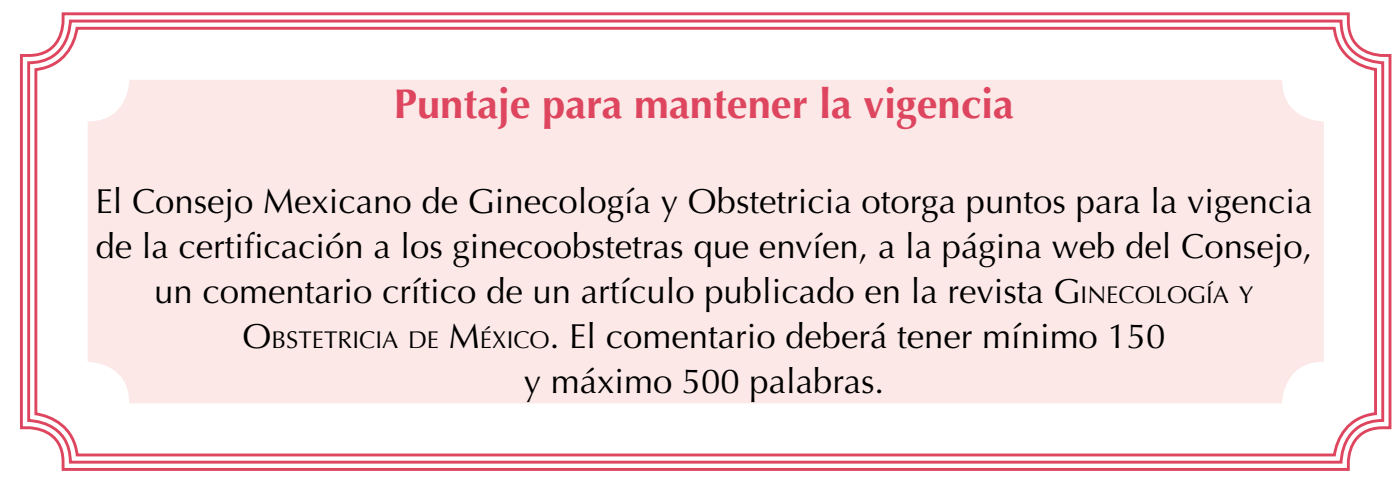

